\title{
El análisis de imágenes como herramienta de monitoreo en la deshidratación de rodajas de banana
}

\author{
Image analysis as a monitoring tool in the dehydration of \\ banana slices
}

José Sebastián Baima1, Pablo Daniel Ribotta ${ }^{1,2,3 *}$ (1)

${ }^{1}$ Universidad Nacional de Córdoba, Instituto Superior de Investigación, Desarrollo y Servicios en Alimentos (ISIDSA), Córdoba - Argentina

${ }^{2}$ Consejo Nacional de Investigaciones Científicas y Técnicas (CONICET), Instituto de Ciencia y Tecnología de los Alimentos Córdoba (ICYTAC), Córdoba - Argentina

${ }^{3}$ Universidad Nacional de Córdoba, Facultad de Ciencias Exactas, Físicas y Naturales, Córdoba - Argentina

*Corresponding Author: Pablo Daniel Ribotta, Consejo Nacional de Investigaciones Científicas y Técnicas (CONICET), Instituto de Ciencia y Tecnología de los Alimentos Córdoba (ICYTAC), Juan Filloy, s/n, Ciudad Universitaria, Código Postal: 5000, Córdoba - Argentina, e-mail: pribotta@agro.unc.edu.ar

Cite as: Baima, J. S., \& Ribotta, P. D. (2019). Image analysis as a monitoring tool in the dehydration of banana slices. Brazilian Journal of Food Technology, 22, e2018231. https://doi.org/10.1590/1981-6723.23118

\begin{abstract}
Resumen
El objetivo de este trabajo fue evaluar los cambios morfométricos, fractales y de color durante la deshidratación de rodajas de banana mediante el uso de análisis de imágenes y la relación de estos parámetros con la cinética de secado. El secado convectivo se realizó entre $75^{\circ} \mathrm{C}$ y $134{ }^{\circ} \mathrm{C}$ a una velocidad de aire de $0,65 \mathrm{~m} \mathrm{~s}^{-1}$. Los resultados mostraron que las imágenes tomadas proporcionaron información cuantitativa de los cambios morfométricos (reducción de área y circularidad, y aumentos del perímetro) por efecto de los tratamientos térmicos. El análisis de la textura fractal detectó cambios en la intensidad de los pixeles, indicando que las superficies resultaron más complejas e irregulares a medida que disminuyó el contenido de humedad del producto. La dimensión fractal de contorno $\left(\mathrm{DF}_{\text {cont }}\right)$ aumentó con el tiempo de secado. En relación al color del producto seco, los parámetros $\mathrm{a}^{*}$ y $\mathrm{b}^{*}$ aumentaron, mientras que la Luminosidad $\left(L^{\star}\right)$ disminuyó, lo cual está asociado al oscurecimiento de las rodajas e influenciado por el aumento de la temperatura. Las micrografías determinaron la presencia de poros que fueron aumentando en cantidad cuando incrementó la temperatura. Los resultados muestran que el análisis de imágenes puede ser empleado como herramienta de monitoreo debido a su versatilidad, bajo costo, rapidez de respuesta, además de no ser una técnica destructiva.
\end{abstract}

Palabras-clave: Secado; Procesamiento de imagen; Banana; Encogimiento; Color; Textura de imagen.

\begin{abstract}
The objective of this work was to evaluate the morphometric, fractal and color changes during the dehydration of banana slices through the use of image analysis. The convective drying was performed between $75^{\circ} \mathrm{C}$ and $134^{\circ} \mathrm{C}$ at an air velocity of $0.65 \mathrm{~m} \mathrm{~s}^{-1}$. Results showed that the images provided quantitative information of the morphometric changes (area and circularity reduction, and perimeter increase) due to the effect of thermal
\end{abstract}


treatments. The analysis of fractal texture detected changes in the intensity of pixels, indicating that the surfaces were more complex and irregular as the moisture content of the product decreased. The fractal dimension of the contour ( $\left.F D_{\text {cont }}\right)$ increased over drying time. Regarding the color of the dried product, the parameters $a^{*}$ and $b^{*}$ increased, whereas the lightness $\left(L^{*}\right)$ decreased, which is associated with the darkening of the slices and influenced by temperature increase. The micrographs determined the presence of pores that increased in quantity when temperature increased. Image analysis can be used as a monitoring tool due to its versatility, low cost, response speed, besides being a non- destructive technique.

Keywords: Drying; Image processing; Banana; Shrinkage; Color; Image texture.

\section{Introducción}

La banana es una de las frutas más consumida a nivel mundial y la alta preferencia de los consumidores se atribuye a su sabor dulce y agradable. La Organización de las Naciones Unidas para la Alimentación y la Agricultura (FAO) coloca al banano en el sexto lugar en la lista de cultivos de mayor producción mundial después de los principales cereales (maíz, trigo, arroz, cebada) y el cultivo de sandía. A pesar de ser un importante cultivo en climas tropicales, su producción alcanza a más de 120 países con un importante aumento interanual. El relevamiento estadístico de la FAO (Organización de las Naciones Unidas para la Alimentación y la Agricultura, 2016) mostró que la producción total de banana a nivel mundial alcanzó 113.280.302 toneladas, siendo India el principal productor mundial con 29.124 .000 toneladas.

Desde el punto de vista biológico, la banana es una de las frutas que presenta las mayores pérdidas por descomposición después de la cosecha ya que es extremadamente perecedera y no permite el uso de la congelación para su conservación (Thakur et al., 2019). Su preservación puede reducir estas pérdidas y aumentar la disponibilidad de la oferta mundial de alimentos (Baini \& Langrish, 2007). La deshidratación es una forma de aprovechar este producto para conservarlo por un periodo de tiempo mayor. El secado convencional con aire caliente es el método más antiguo utilizado en la conservación de alimentos y ha sido ampliamente aplicado para el secado de bananas (Monteiro et al., 2016).

En los últimos años, los métodos de análisis de imágenes se han convertido en una herramienta importante en los procesos de inspección, selección y producción de alimentos debido a su capacidad de automatización y de ser una técnica no destructiva, particularmente en relación a la producción de frutas y verduras (VélezRivera et al., 2014). La técnica de análisis de imágenes permite valorar cambios que ocurren durante un proceso en base a las características que se extraen desde las imágenes.

El objetivo de este trabajo fue analizar el proceso de deshidratación de rodajas de banana mediante análisis de imágenes y geometría de fractales y relacionar los resultados con la cinética de secado y el desarrollo de color.

\section{Materiales y métodos}

\subsection{Materia prima y preparación de las muestras}

Se trabajó con bananas de la marca Banana Tropical de origen ecuatoriano (Musa Cavendish). La madurez se determinó de acuerdo a la escala de Von Loesecke con grado de maduración 5 (fruto amarillo con puntas verdes). Las bananas fueron peladas y se seleccionó la parte central (descartando los extremos) de cada fruta y luego se cortaron en rebanadas de $3 \mathrm{~mm}$ de espesor. Las muestras se sumergieron en una solución con $10 \mathrm{~g} / \mathrm{L}$ de ácido ascórbico y $10 \mathrm{~g} / \mathrm{L}$ de ácido cítrico durante 1 minuto antes del secado lo cual fue adoptado sobre la base de una práctica utilizada en la industria alimentaria según describe Pan et al. (2008). 


\subsection{Deshidratación}

Las rodajas fueron deshidratadas en horno de convección forzada Philco HE-PH40W a diferentes temperaturas. Las temperaturas reales de trabajo se midieron utilizando termocuplas tipo K. Los rangos de temperatura de deshidratación fueron: $75^{\circ} \mathrm{C}$ a $86{ }^{\circ} \mathrm{C}(\mathrm{T} 1), 93{ }^{\circ} \mathrm{C}$ a $99^{\circ} \mathrm{C}(\mathrm{T} 2)$ y $127{ }^{\circ} \mathrm{C}$ a $134{ }^{\circ} \mathrm{C}(\mathrm{T} 3)$.

Cada lote de ensayo estuvo conformado por una bandeja con 35 rodajas de banana distribuidas homogéneamente en la bandeja de secado. Se realizaron ensayos preliminares para determinar los tiempos de secado a cada temperatura. A diferentes tiempos de secado (Tabla 1) y mediante ensayos independientes según se registró la pérdida de peso de cada lote con una balanza analítica (Ohaus, TA 150) y se tomaron muestras de rodajas de banana para su análisis. Los tratamientos a cada temperatura se realizaron al menos por duplicado.

Tabla 1. Esquema de los ensayos de deshidratación y tiempos de toma de muestra y secado para cada temperatura.

\begin{tabular}{ccccccccccccc}
\hline Temperatura de secado & \multicolumn{10}{c}{ Tiempo [min] } \\
\hline $\mathrm{T} 1$ & 0 & 15 & 30 & 45 & 60 & 90 & 120 & 150 & 180 & 210 \\
$\mathrm{~T} 2$ & 0 & 10 & 15 & 20 & 25 & 30 & 35 & 40 & 50 & 60 & 65 \\
$\mathrm{~T} 3$ & 0 & 5 & 10 & 15 & 20 & 25 & 28 & 30 & & \\
\hline $\mathrm{T} 1=75^{\circ} \mathrm{C}$ a $86^{\circ} \mathrm{C} ; \mathrm{T} 2=93^{\circ} \mathrm{C}$ a $99^{\circ} \mathrm{C} ; \mathrm{T} 3=127^{\circ} \mathrm{C}$ a $134^{\circ} \mathrm{C}$.
\end{tabular}

El contenido de humedad inicial de las muestras se analizó de acuerdo a la metodología propuesta por Association of Official Analytical Chemists (1995). Los ensayos se realizaron por duplicado.

\subsection{Difusividad efectiva y energía de activación}

Para determinar el coeficiente de difusión efectivo $\left(\mathrm{D}_{\mathrm{f}}\right)$ se utilizó la ecuación de la segunda ley de Fick (Ecuación 1), considerando tiempos largos de secado y geometría plana en una dimensión (Baini \& Langrish, 2007).

$$
\mathrm{MR}=\frac{\mathrm{X}-\mathrm{X}_{\mathrm{e}}}{\mathrm{X}_{0}-\mathrm{X}_{\mathrm{e}}}=\frac{8}{\pi^{2}} \exp ^{\left(\frac{\mathrm{D}_{\mathrm{f}} \pi^{2} \mathrm{t}}{41^{2}}\right)}
$$

donde MR es la razón de humedad; $\mathrm{X}$ es la humedad a cada tiempo intermedio de secado de la Tabla 1 ( $\mathrm{kg}$ agua/kg sólido seco); $\mathrm{X}_{\mathrm{e}}$ es la humedad de equilibrio ( $\mathrm{kg}$ agua $/ \mathrm{kg}$ sólido seco); $\mathrm{X}_{0}$ es la humedad inicial (kg agua/kg sólido seco); $\mathrm{t}$ es el tiempo (s); 1 es el semiespesor de la lámina (m) y $\mathrm{D}_{\mathrm{f}}$ es el coeficiente de difusión efectivo $\left(\mathrm{m}^{2} \mathrm{~s}^{-1}\right)$. El $\mathrm{D}_{\mathrm{f}}$ para cada temperatura se calculó a partir de la pendiente de la línea recta obtenida al graficar el Ln (MR) en función del tiempo de secado.

La influencia de la temperatura de secado sobre la difusividad del agua desde el interior del alimento hacia su superficie se analizó por la ecuación de Arrhenius (Ecuación 2).

$D_{\mathrm{f}}=\mathrm{D}_{0} \exp \left(-\frac{\mathrm{E}_{\mathrm{a}}}{\mathrm{RT}}\right)$

donde $\mathrm{D}_{0}$ es el factor de Arrhenius $\left(\mathrm{m}^{2} \mathrm{~s}^{-1}\right) ; \mathrm{E}_{\mathrm{a}}$ es la energía de activación $\left(\mathrm{kJ} \mathrm{mol}^{-1}\right)$; $\mathrm{T}$ es la temperatura absoluta de secado $(\mathrm{K})$ y R es la constante universal de los gases $\left(8,314 \times 10^{-3} \mathrm{~kJ} \mathrm{~mol}^{-1} \mathrm{~K}^{-1}\right)$. Representando gráficamente el $\operatorname{Ln}\left(\mathrm{D}_{\mathrm{f}}\right)$ en función de $1 / \mathrm{T}(\mathrm{K})$ se obtiene una línea recta, de cuya pendiente se extrae la energía de activación $\left(E_{a}\right)$ y su ordenada al origen corresponde al factor de Arrhenius $\left(D_{0}\right)$.

\subsection{Adquisición y análisis de imágenes}

Se obtuvieron imágenes de las rodajas de banana fresca y deshidratadas a diferentes tiempos y temperaturas (según Tabla 1). Las imágenes se obtuvieron con cámara digital Panasonic Lumix, modelo DMC-TZ3 con iluminación fija e igual en cada ensayo. Las fotografías se tomaron sin flash, sin zoom, con 
autodisparador, en modo macro y con ajuste en "modo fino". Se colocó una regla milimetrada que sirvió como referencia para calibrar las imágenes y convertir pixeles en centímetros. Las imágenes RGB en formato de archivo JPG se analizaron con el programa ImageJ versión $1.49 \mathrm{~m}$, de dominio público creado por National Institutes of Health (USA).

\subsection{Análisis morfométrico}

El análisis morfométrico se realizó tomando 15 rodajas por cada lote ubicadas en la parte central de la bandeja de secado. Las imágenes RGB se convirtieron a 8-bits (0 negro, 255 blanco) y posteriormente se creó una imagen binaria que permitió evaluar cambios en los parámetros morfométricos de área ( $A$, número total de pixeles que cae dentro del contorno del elemento, multiplicado por el área de un pixel), perímetro ( $P$, número total de pixeles pertenecientes al contorno del objeto), circularidad $(C$, similitud del objeto comparado con un referente perfectamente circular y varía entre cero y uno, siendo uno un círculo perfecto) y diámetro de Feret ( $d F$, se define como la mayor distancia de separación entre dos pixeles tomados a orientaciones diferentes).

\subsection{Análisis de textura y fractal}

Se tomaron 5 rodajas de la parte central de las bandejas por cada lote y se seleccionó un área de interés (ROI) de $87 \times 255$ pixeles que abarcó desde un extremo al otro de cada rodaja, pasando por la parte central. Las imágenes RGB se convirtieron a 8-bits y se analizaron mediante el algoritmo de la Matriz de Coocurrencia en escala de grises (GLCM, Gray Level Co-Ocurrence Matrix) para determinar las características de textura superficial a partir de imágenes en escala de grises. Este algoritmo es de segundo orden y considera la relación entre parejas de pixeles. Se evaluaron los siguientes cuatro parámetros texturales, de los catorce que se puede obtener según Ranjit et al. (2016).

Energía (ASM, Angular Second Moment), es una medida de la homogeneidad local de una imagen. Presenta altos valores cuando la frecuencia de aparición de las parejas de pixeles en la matriz de co-ocurrencia tiene la misma intensidad de nivel de gris.

Contraste $(C O N)$, es una medida de la variación local del nivel de gris. Aumenta a medida que el nivel de gris de la pareja de pixeles se aleja hacia los extremos de la escala en una imagen de 8 bits.

Homogeneidad (IDM, Inverse Difference Moment), es una medida de la diferencia en la intensidad de gris entre pixeles vecinos.

Entropía (ENT), es una variable que está relacionada con el orden o desorden que tiene una imagen y es usada para caracterizar la textura de las imágenes. Imágenes complejas tendrán altos valores de entropía (Mendoza et al., 2007).

En la Tabla 2 se presentan las ecuaciones de los parámetros texturales del algoritmo de GLCM.

Paralelamente se utilizó el Algoritmo Modificado de Conteo Diferencial de Cajas (SDBC, Shifting Differential Box Counting) para determinar los valores de la dimensión fractal de textura de las imágenes, la que es posible utilizarla para caracterizar la textura de las superficies y expresarla como una medida de la rugosidad o irregularidad que presenta la superficie de una imagen (Quevedo et al., 2008). Este algoritmo se calculó a partir de un gráfico de intensidad de superficie generado a partir de los pixeles de la imagen $2 \mathrm{D}$ en escala de grises (coordenadas $x, y$ ) y sus intensidades en el eje $z$. La configuración del tamaño de cajas se consideró como "automático" que es la opción predeterminada por el programa ImageJ y comprendió tamaños entre 10 y 50 pixeles cuadrados. El valor de la dimensión fractal de superficie $\left(\mathrm{DF}_{\text {sup }}\right)$ se obtuvo de la pendiente de la relación logarítmica del número de cajas $(\log N)$ en función del logaritmo del tamaño de las cajas $(\log r)$. Los valores de dimensión fractal oscilan entre 2 y 3 . Las superficies lisas, sin rugosidad, presentan valores numéricos próximos a $2\left(\mathrm{DF}_{\text {sup }}>2\right)$, mientras que las superficies altamente rugosas 
presentan un incremento en los valores de la dimensión fractal (Santacruz-Vázquez et al., 2008). Se analizó 5 rodajas por cada lote a cada uno de los tiempos de secado de la Tabla 1.

La dimensión fractal de contorno ( $\mathrm{DF}_{\text {cont }}$ ) también se evaluó utilizando el Algoritmo Modificado de Conteo Diferencial de Cajas (SDBC) sobre imágenes de los contornos de las rodajas. Esta herramienta permitió determinar cuan irregular fue el borde de las rodajas ya que su forma, como consecuencia de la deformación durante la deshidratación, no pudo ser explicada por medio del uso de la geometría tradicional. Cuanto más irregular sea el contorno del objeto mayor será su $\mathrm{DF}_{\text {cont. }}$ Se trabajó con 5 rodajas por lote - las mismas que se utilizó en $G L C M$ - tomando un ROI de $368 \times 379$ pixeles que abarcó la rodaja completa. Las imágenes RGB se convirtieron a 8-bits, se creó una imagen binaria, se llenó el objeto correspondiente a la rodaja y se trazó el contorno.

Tabla 2. Ecuaciones de los parámetros texturales del algoritmo de GLCM (Ecuación 3, 4, 5 y 6).

\begin{tabular}{ccccc}
\hline Parámetro textural & Ecuación & Parámetro textural & Ecuación \\
\hline Energía $(A S M)$ & $\sum_{\mathrm{i}, \mathrm{j}=0}^{\mathrm{N}-1} \mathrm{P}_{\mathrm{i}, \mathrm{j}}^{2}$ & 3 & Homogeneidad $(I D M)$ & $\sum_{\mathrm{i}, \mathrm{j}=0}^{\mathrm{N}-1} \frac{\mathrm{P}_{\mathrm{i}, \mathrm{j}}}{1+(\mathrm{i}-\mathrm{j})^{2}}$ \\
Contraste $(C O N)$ & $\sum_{\mathrm{i}, \mathrm{j}=0}^{\mathrm{N}-1} \mathrm{P}_{\mathrm{i}, \mathrm{j}}(\mathrm{i}-\mathrm{j})^{2}$ & 4 & Entropía $(E N T)$ & $\sum_{\mathrm{i}, \mathrm{j}=0}^{\mathrm{N}-1}-\mathrm{P}_{\mathrm{i}, \mathrm{j}} \ln \mathrm{P}_{(\mathrm{i}, \mathrm{j})}$ \\
\hline
\end{tabular}

$i, j=$ intensidades de nivel de gris de cada uno de los pixeles que forman la pareja, comprendidos entre 0 (negro) y 255 (blanco); $\mathrm{N}=$ número de niveles de gris posibles ( 256 en una fotografía de 8 bits); $P_{i, j}=$ probabilidad de aparición de la pareja de pixeles con intensidades de gris $i$ y $j$ respectivamente, a una distancia de separación entre pixeles igual a 1 y con un ángulo de desplazamiento de $0^{\circ}$.

\subsection{Análisis de color y pardeamiento de las rodajas}

Para el análisis de los cambios de color durante el proceso de deshidratación se utilizó el submenú Color Inspector 3D (v2.3) dentro del menú Plugins del ImageJ, seleccionando el espacio de color CIELab a partir del cual se obtuvieron los parámetros $\mathrm{L}^{*}, \mathrm{a}^{*} \mathrm{y} \mathrm{b}^{*}$. Se tomaron 5 ROIs $(87 \times 255$ pixeles $)$ y se realizó un promedio de color de los pixeles de la imagen, resultando en una superficie de una sola tonalidad a partir de la cual se obtuvieron los parámetros. Los resultados se presentan como el promedio de los 5 ROIs. La diferencia de color ( $\triangle E$, Ecuación 7) se utilizó para describir el cambio de color de las rodajas durante el tratamiento térmico. Esta ecuación tiene en cuenta el cambio en cada uno de los colores descrito por los parámetros $\mathrm{L}^{*}, \mathrm{a}^{*} \mathrm{y} \mathrm{b}^{*}$, y puede ser más aplicable que los tres parámetros de color de forma individual para evaluar el efecto de las condiciones de secado en estos frutos (Baini \& Langrish, 2009; Atares et al., 2011).

$\Delta \mathrm{E}=\sqrt{\left(\mathrm{L}^{*}-\mathrm{L}_{0}^{*}\right)^{2}+\left(\mathrm{a}^{*}-\mathrm{a}_{0}^{*}\right)^{2}+\left(\mathrm{b}^{*}-\mathrm{b}_{0}^{*}\right)^{2}}$

$\mathrm{L}^{*}$ representa la Luminosidad $\left(\mathrm{L}^{*}=0\right.$, negro y $\mathrm{L}^{*}=100$, blanco); $\mathrm{a} *$ indica la posición entre verde y rojo (valores negativos indican verde mientras que valores positivos indican rojo); $\mathrm{y} \mathrm{b}^{*}$ indica la posición entre azul y amarillo (valores negativos indican azul mientras que valores positivos indican amarillo). $\mathrm{L} *_{0}, \mathrm{a}_{0} \mathrm{y} \mathrm{b}_{0}$ corresponde a valores iniciales, mientras que $\mathrm{L}^{*}, \mathrm{a}^{*} \mathrm{y} \mathrm{b}^{*}$ corresponde a los tiempos de secado (Tabla 1).

El índice de pardeamiento (BI, “Browning Index”) representa la pureza del color marrón y se ha reportado como un importante parámetro en procesos donde el pardeamiento enzimático y no enzimático tiene lugar (Pu et al., 2018). Se calculó utilizando la Ecuación 8.

$\mathrm{BI}=\frac{100(\mathrm{x}-0,31)}{0,172}$

donde $x$, está representado en la Ecuación 9.

$\mathrm{x}=(\mathrm{a} *+1,75 \mathrm{~L} *) /\left(5,645 \mathrm{~L} *+\mathrm{a}^{*}-3,012 \mathrm{~b} *\right)$ 


\subsection{Microscopía electrónica de barrido (SEM)}

La ultra estructura de las rodajas de banana deshidratadas fue analizada mediante microscopía electrónica de barrido. Se obtuvieron trozos de rodajas deshidratadas, se montaron sobre un porta muestras y se recubrieron con una fina capa de elemento conductor (oro) mediante un sistema de recubrimiento por pulverización catódica. Para realizar las observaciones se utilizó un microscopio de barrido electrónico FESEM (Carl Zeiss- Sigma, Alemania) bajo condiciones de alto vacío (10 a 4 Pa) a un voltaje de aceleración de $5 \mathrm{kV}$. Las fotografías se tomaron utilizando un software de captura automática de imágenes.

\subsection{Análisis estadístico}

Se utilizó el software estadístico InfoStat (Facultad de Ciencias Agropecuarias, Universidad Nacional de Córdoba) con el cual se analizaron los datos obtenidos mediante análisis de varianza (ANOVA). Los resultados fueron comparados con el método DGC y las diferencias significativas fueron consideradas cuando $p<0,05$. La prueba DGC constituye un método particionante (sin solapamiento de letras entre las medias) recomendable para comparar cinco o más medias (Di Rienzo et al., 2002).

\section{Resultados y discusión}

\subsection{Cambios en el contenido de humedad de las rodajas}

La humedad inicial de las muestras frescas de Musa cavendish fue de $78,11 \% \pm 0,6 \%$, lo que equivale a $3,57 \pm 0,13 \mathrm{~kg}$ agua $/ \mathrm{kg}$ sólido seco. Similares contenidos de humedad inicial fueron informados por otros autores (Chua et al., 2001; Leite et al., 2007). La Figura 1A muestra la disminución de la humedad de las piezas de banana durante los tratamientos térmicos. Se observó que cuanto mayor fue la temperatura del aire, más rápidamente se secó el producto y más corto fue el tiempo de procesamiento. El contenido de humedad final fue inferior a $0,04 \mathrm{~kg}$ agua $/ \mathrm{kg}$ sólido seco para T1 e inferior a $0,02 \mathrm{~kg}$ agua $/ \mathrm{kg}$ sólido seco para T2 y T3.

En la Figura 1B se muestran las curvas de velocidad para las tres temperaturas de secado de las rodajas de banana. En ellas se observa una clara dependencia de la velocidad con la temperatura de aire, aumentando la pendiente de las mismas, a medida que incrementó el aporte de calor sobre las muestras. Además, se puede observar que el secado se llevó a cabo bajo condiciones de velocidad decreciente, lo cual muestra que el proceso es controlado por la difusión de la humedad desde el interior del fruto hacia la superficie como ocurrió en el secado de rodajas de calabaza (Doymaz, 2007) y semillas de tomate (Sogi et al., 2003).

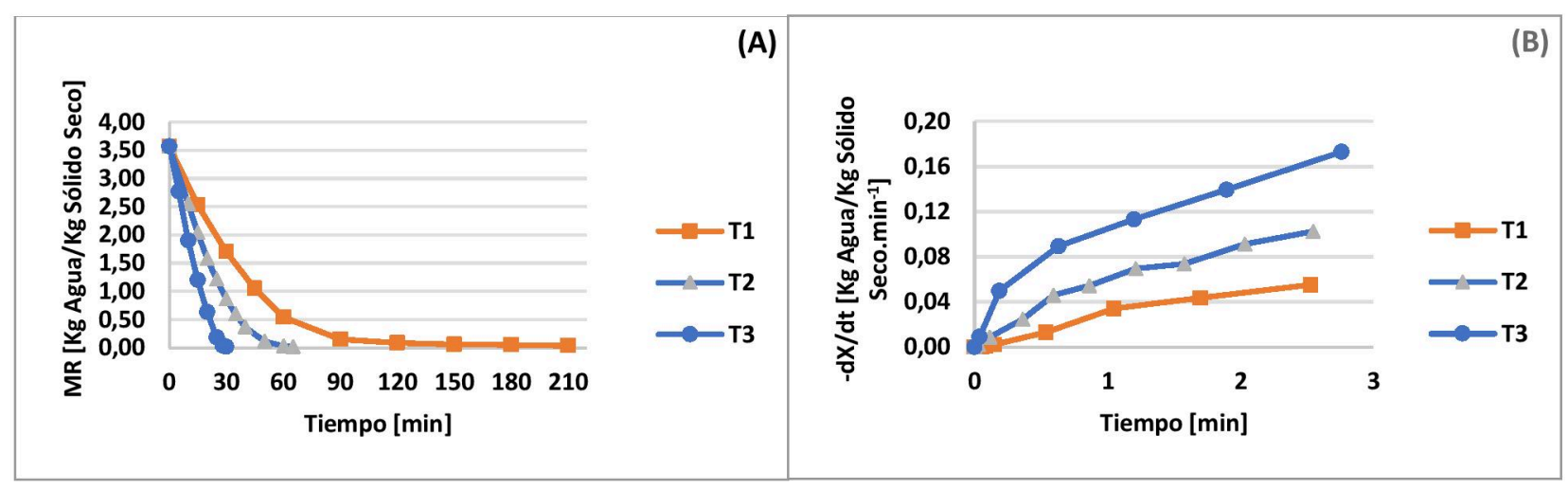

Figura 1. Efecto de la temperatura del aire sobre el contenido de humedad (A) y la velocidad de secado (B) de rodajas de banana deshidratadas por convección. 


\subsection{Difusividad efectiva y energía de activación}

Los valores de los coeficientes de difusión se observan en la Tabla 3. Estos estuvieron dentro de los datos encontrados en la bibliografía para la mayoría de los alimentos (entre $10^{-9}$ a $10^{-11} \mathrm{~m}^{2} \mathrm{~s}^{-1}$, según Rao et al., 2005). Los coeficientes aumentaron significativamente cuando aumentó la temperatura demostrando la existencia de una menor resistencia interna del alimento al aumento de la temperatura durante el secado.

Tabla 3. Coeficientes de difusión efectivo obtenidos para las diferentes temperaturas de secado.

\begin{tabular}{cc}
\hline Temperatura de secado & $\mathbf{D}_{\mathbf{f}}\left[\mathbf{x} \mathbf{1 0}^{-\mathbf{1 0}} \mathbf{~ m}^{\mathbf{2}} \mathbf{s}^{-1}\right]$ \\
\hline $\mathrm{T} 1$ & $5,4713 \pm 0,0001^{\mathrm{a}}$ \\
$\mathrm{T} 2$ & $8,6630 \pm 0,6448^{\mathrm{b}}$ \\
$\mathrm{T} 3$ & $15,0462 \pm 0,6448^{\mathrm{c}}$ \\
\hline
\end{tabular}

Medias con una letra diferente en la misma columna son significativamente diferentes $(p<0,05)$. T1 $=75{ }^{\circ} \mathrm{C}$ a $86{ }^{\circ} \mathrm{C}$; $\mathrm{T} 2=93{ }^{\circ} \mathrm{C}$ a $99{ }^{\circ} \mathrm{C}$; $\mathrm{T} 3=127^{\circ} \mathrm{C}$ a $134^{\circ} \mathrm{C}$.

El valor obtenido de energía de activación fue de $23,66 \mathrm{~kJ} \mathrm{~mol}^{-1}$ y representó la mínima energía requerida por el agua del alimento para ser eliminada durante el proceso de secado. El rango de 12,7 a $110 \mathrm{~kJ} \mathrm{~mol}^{-1}$ comprende energías de activación en la que se encuentran la mayoría de los productos agroalimentarios, como por ejemplo manzana (Horuz et al., 2017), zanahoria (Doymaz, 2004), germen de trigo (Gili et al., 2018) y espinacas (Doymaz, 2009). El factor de Arrhenius presentó un valor de $1,79 \times 10^{-6} \mathrm{~m}^{2} \mathrm{~s}^{-1}$.

\subsection{Análisis morfométrico}

La Figura 2 muestra la variación de los parámetros morfométricos. El área $(A)$ inicial promedio de las rodajas determinada por análisis de imágenes estuvo comprendida en el rango de 6,85 a $7,42 \mathrm{~cm}^{2}$. El proceso de secado provocó el encogimiento de las rodajas de banana- fenómeno esperado en este tipo de procesos resultando en un área final entre 6,16 y $6,47 \mathrm{~cm}^{2}$. La reducción de área en $\mathrm{T} 1$ fue mayor (13\%) debido a que la deshidratación fue más lenta y provocó un efecto de contracción más acentuado. Por el contrario, cuando la deshidratación fue rápida (T2 y T3) la estructura se rigidizó más rápidamente y mostró menor encogimiento y por consecuencia mayor área de rodaja (Figura $2 \mathrm{~A}$ ).

El perímetro $(P)$ (Figura 2B) presentó bordes bien definidos al inicio del secado y valores entre 10,05 y 10,48 cm. En los primeros minutos, se observó una leve disminución que acompañó a la reducción del área provocada por efecto de la temperatura, manteniendo bordes lisos y bien delimitados. Posteriormente, la deshidratación produjo deformaciones sobre el contorno de la fruta y el perímetro aumentó continuamente $(p<0,05)$, resultando en valores finales entre 11,35 y $12,29 \mathrm{~cm}$.

Por otra parte, las rodajas frescas mostraton valores de circularidad $(C)$ altos al inicio del secado $(0,84-0,86)$ (Figura 2C) y una reducción comprendida entre un 30\% a 33\% debido a su deshidratación, lo que originó una forma alargada en el producto seco como consecuencia de deformaciones en la estructura de la fruta. Este parámetro también estuvo influenciado por la temperatura de secado, ya que a T3 la disminución de la circularidad ocurrió más rápidamente con tiempos de procesamiento considerablemente menores.

El diámetro de Feret $(d F)$ (Figura 2D) disminuyó con el tiempo de procesamiento demostrando que la estructura de la fruta cambió y que el agua eliminada provocó el encogimiento de las rodajas durante el secado. 


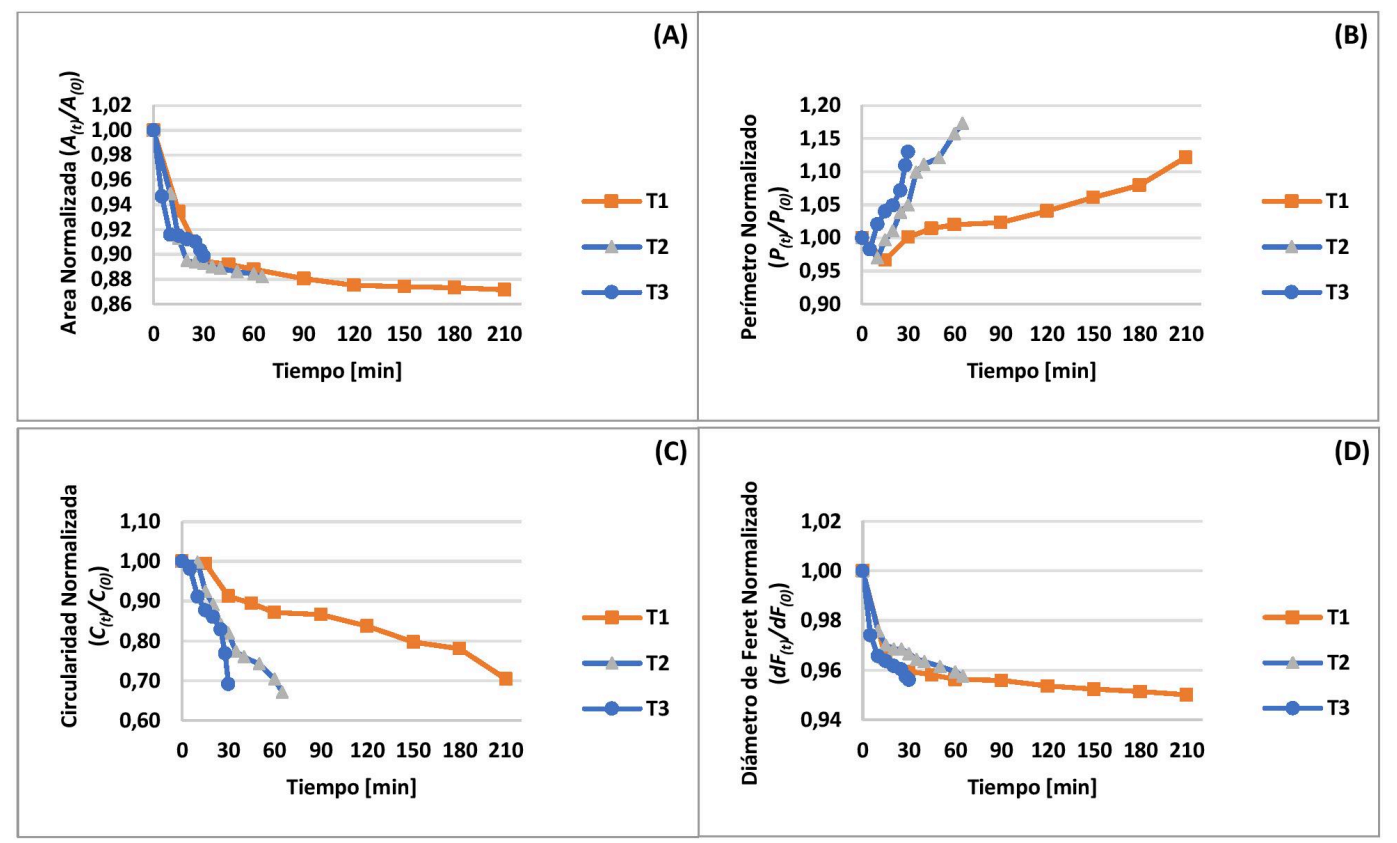

Figura 2. Efecto de la temperatura de deshidratación sobre los parámetros morfométricos: (A) Área; (B) Perímetro; (C) Circularidad; (D) Diámetro de Feret $\left(\mathrm{T} 1=75^{\circ} \mathrm{C}\right.$ a $86{ }^{\circ} \mathrm{C}$; $\mathrm{T} 2=93{ }^{\circ} \mathrm{C}$ a $99{ }^{\circ} \mathrm{C}$; T3 $=127{ }^{\circ} \mathrm{C}$ a $\left.134{ }^{\circ} \mathrm{C}\right)$.

Durante el secado convectivo, el agua que se elimina produce un desequilibrio entre la presión interna del material y la presión externa, generando tensiones que dan lugar a la contracción o al colapso del material, cambios en la forma y ocasionalmente el agrietamiento del producto (Mayor \& Sereno, 2004).

\subsection{Análisis de textura y fractal}

La variación de la energía y la homogeneidad en función del tiempo de secado se muestra en la Figura $3 \mathrm{~A}$ y B. El análisis $G L C M$ determinó que la energía $(A S M)$ y la homogeneidad (IDM) mostraron tendencias similares, disminuyendo ambas en función del tiempo de secado. Los altos valores encontrados al inicio del tratamiento (rodajas frescas, $\mathrm{t}=0 \mathrm{~min}$ ) se debieron a que las imágenes de 8 bits tuvieron una alta similitud entre parejas de pixeles, con una escasa diferencia en los tonos de gris que presentaron. Además, al estar uniformemente distribuidas y con una alta frecuencia de aparición, reflejaron una superficie lisa, uniforme y homogénea. Durante el proceso de secado, se modificó la textura superficial de la rodaja debido a la evaporación de agua, y la uniformidad textural comenzó a decaer, lo que se reflejó en pixeles que presentaron distinta intensidad en sus niveles de gris. En el secado de discos de manzana, realizado por Fernández et al. (2005), se encontró que la disminución de la homogeneidad se debió a la pérdida en la uniformidad textural de las muestras.

Los valores de entropía (ENT) y contraste $(C O N)$ (Figura 3C y D) aumentaron a medida que avanzó el secado. La entropía es un parámetro opuesto a la energía y como tal manifestó un comportamiento contrario. Su aumento dejó en evidencia que los snacks presentaron imágenes más complejas respecto de las imágenes tomadas a las rebanadas frescas. A su vez, y como sucedió con otros parámetros, una mayor temperatura de secado aceleró el cambio de entropía. En el contexto del procesamiento de imágenes, este aumento reflejó que las parejas de pixeles presentaron un desorden en la distribución de los niveles de gris que aportó información de lo ocurrido en el secado y mostró, como se puedo observar visualmente, que las rodajas adquirieron una superficie menos uniforme y de textura compleja. Los altos valores de entropía se evidencian por la elevada propagación de pixeles de variada intensidad y en forma uniforme sobre la imagen espectral (Park \& Chen, 2001), demostrando un mayor desorden y deformación superficial consecuencia del proceso de deshidratación. 


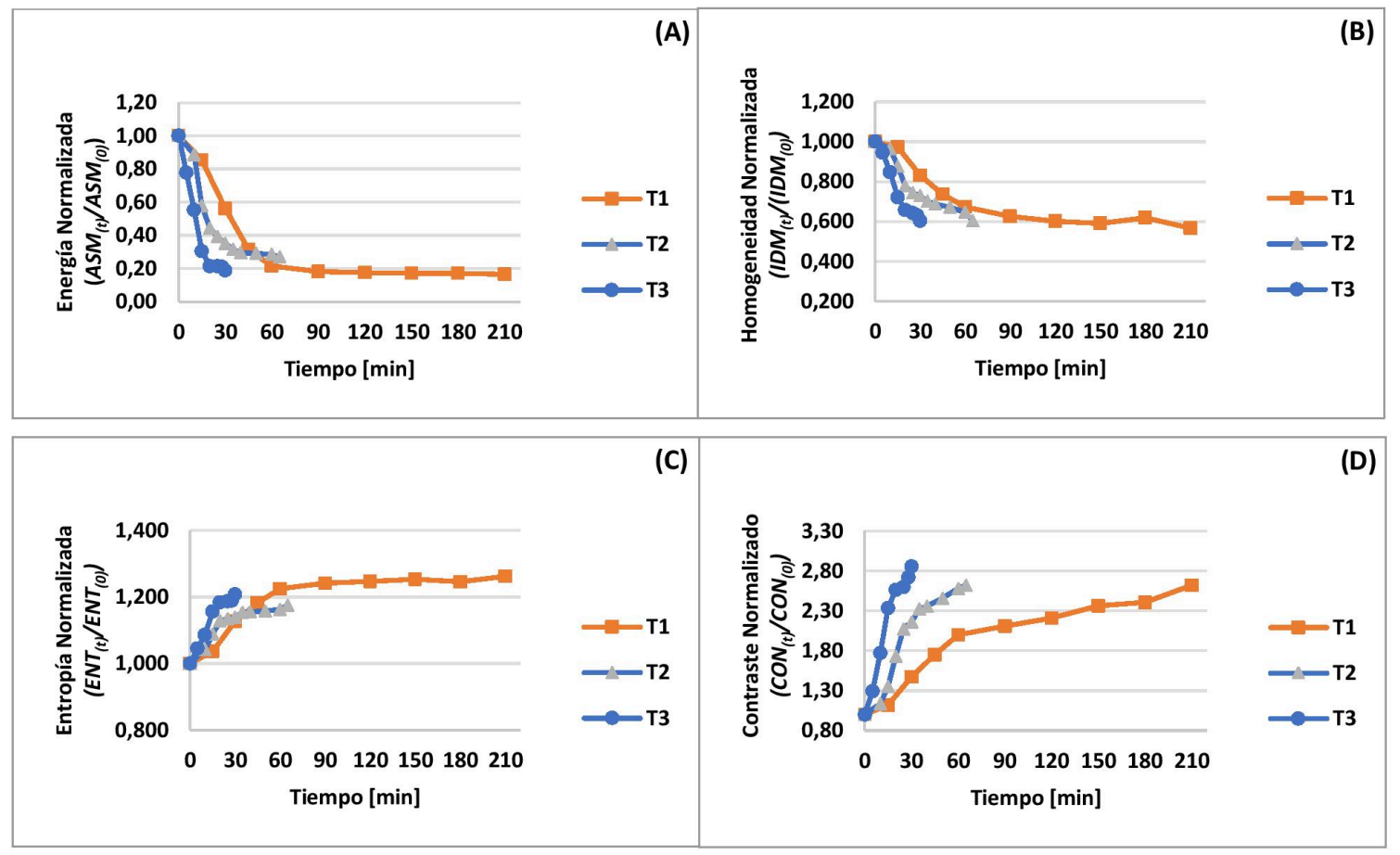

Figura 3. Efecto de la temperatura de deshidratación sobre la energía (A), la homogeneidad (B), la entropía (C) y el contraste (D) para diferentes condiciones de secado $\left(\mathrm{T} 1=75^{\circ} \mathrm{C}\right.$ a $86{ }^{\circ} \mathrm{C}$; $\mathrm{T} 2=93{ }^{\circ} \mathrm{C}$ a $99{ }^{\circ} \mathrm{C}$; $\mathrm{T} 3=127{ }^{\circ} \mathrm{C}$ a $\left.134^{\circ} \mathrm{C}\right)$.

Por otra parte, el contraste mostró un comportamiento bien definido a cada temperatura. La pendiente de la gráfica manifiesta la rapidez con la que cambió este parámetro. Al igual que la homogeneidad, el contraste es una medida de la variación local del nivel de gris de pixeles vecinos, por lo que su aumento se atribuyó a la diferente intensidad que presentaron los pixeles de la imagen. La modificación del contraste estuvo asociada al cambio de color en la superficie de las rodajas durante el secado, con pixeles que presentaron intensidad de gris más cercana a cero (aumento del negro), lo cual significó un mayor oscurecimiento provocado por el pardeamiento de las muestras.

En la Tabla 4 se muestra la correlación de Pearson entre la humedad y los parámetros de textura de las imágenes. Los resultados indicaron que los coeficientes tuvieron una alta correlación y demostraron que las propiedades evaluadas están fuertemente asociadas.

Tabla 4. Correlación de Pearson entre humedad y los parámetros fractales.

\begin{tabular}{|c|c|c|c|c|c|}
\hline & $\begin{array}{c}\text { Parámetro } \\
\text { textural }\end{array}$ & $\begin{array}{c}\text { Humedad } \\
{[\mathrm{kg} \text { agua }(\mathrm{t}) / \mathrm{kg} \text { agua }(0)]}\end{array}$ & $A S M$ & CON & $I D M$ \\
\hline \multirow{4}{*}{$\mathrm{T} 1$} & $A S M$ & 0,99 & & & \\
\hline & CON & $-0,96$ & $-0,94$ & & \\
\hline & $I D M$ & 0,98 & 0,98 & $-0,98$ & \\
\hline & ENT & $-0,99$ & $-1,00$ & 0,96 & $-0,99$ \\
\hline \multirow{4}{*}{$\mathrm{T} 2$} & $A S M$ & 0,96 & & & \\
\hline & $C O N$ & $-0,98$ & $-0,94$ & & \\
\hline & $I D M$ & 0,98 & 0,97 & $-0,99$ & \\
\hline & ENT & $-0,97$ & $-0,99$ & 0,95 & $-0,98$ \\
\hline \multirow{4}{*}{$\mathrm{T} 3$} & $A S M$ & 0,98 & & & \\
\hline & CON & $-0,99$ & $-0,99$ & & \\
\hline & $I D M$ & 0,99 & 0,99 & $-1,00$ & \\
\hline & ENT & $-0,99$ & $-0,99$ & 1,00 & $-1,00$ \\
\hline
\end{tabular}

En todos los casos valores $p<0,01 . A S M=$ energia; $C O N=$ contraste; $I D M=$ homogeneidade; $E N T=$ entropía. 
El análisis de los parámetros $G L C M$ determinó que la entropía fue el parámetro que mostró mayor nivel de correlación con la pérdida de humedad en el producto. El desorden de las imágenes, que se reflejó en una mayor cantidad de niveles de grises a medida que avanzó el tiempo de secado, guardó una alta relación con la disminución de humedad en el producto.

En la Figura 4 se muestra la topografía superficial de las imágenes y los valores de dimensión fractal de superficie $\left(\mathrm{DF}_{\text {sup }}\right)$ a distintos tiempos de secado de cada temperatura. El análisis de textura fractal detectó cambios en la intensidad de los pixeles indicando que la superficie de la banana resultó más rugosa e irregular a medida que disminuyó su contenido de humedad $(p<0,05)$. Al inicio del secado, la $\mathrm{DF}_{\text {sup }}$ estuvo comprendida entre 2,20 y 2,25 , asociado a una menor complejidad topográfica expresada por las menores diferencias de los niveles de grises de los pixeles, y por lo tanto, atribuidos a una superficie más lisa y homogénea. La transferencia de calor durante el secado provocó la disminución de la humedad en el producto y sugirió cambios a nivel superficial. Estas modificaciones provocaron un aumento de la complejidad superficial, fenómeno marcado por un comportamiento no uniforme. Los valores finales estuvieron comprendidos entre 2,32 y 2,37 . $\mathrm{La} \mathrm{DF}_{\text {sup }}$ y la entropía presentaron un comportamiento opuesto a la energía y la homogeneidad. La relación entre estos parámetros demostró que el procesamiento provocó un cambio en la complejidad superficial del producto que pudo ser evidenciado por análisis de textura de imágenes.

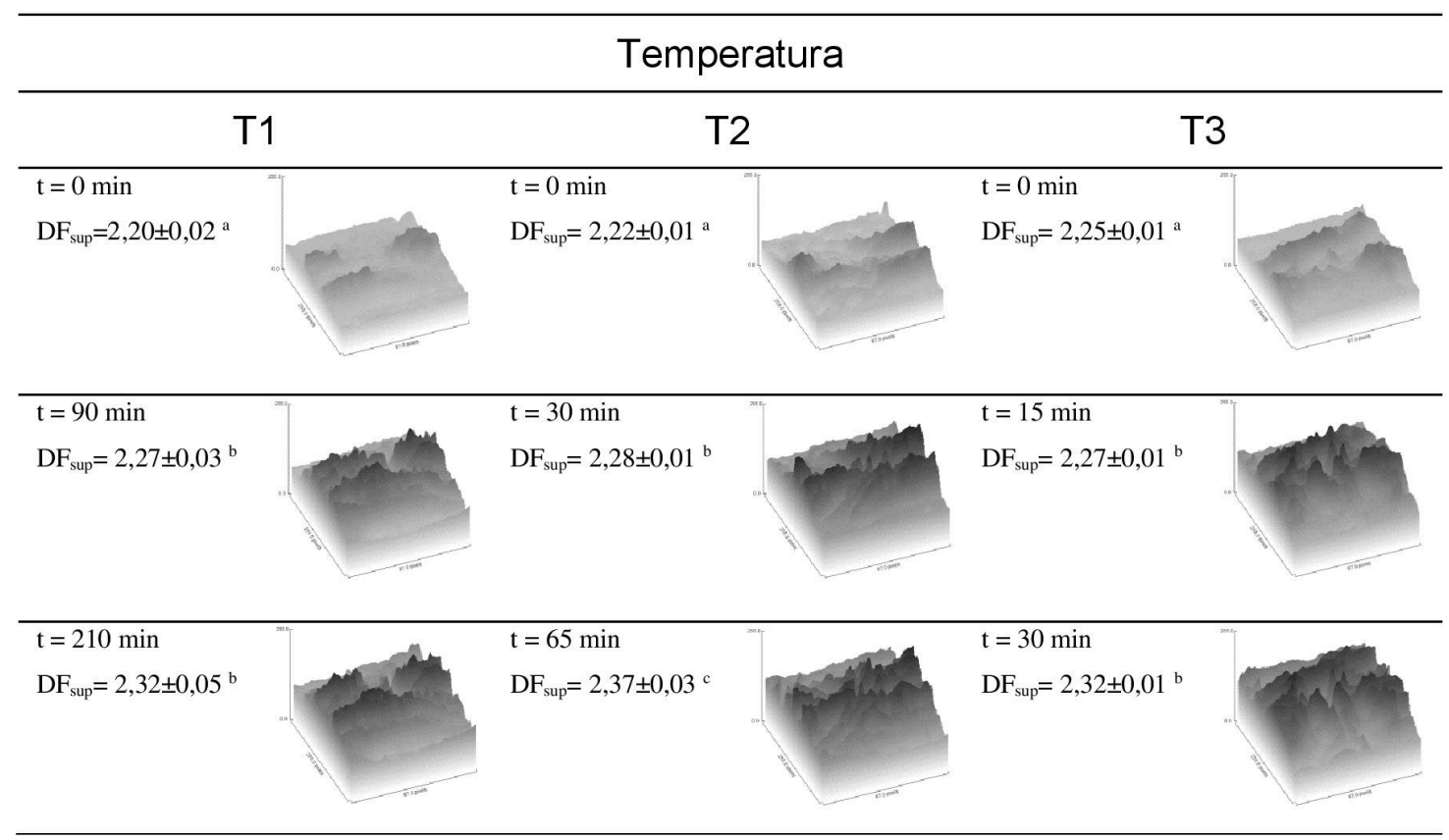

Figura 4. Topografía superficial $3 \mathrm{D}$ de rodajas de banana y sus correspondientes valores de $\mathrm{DF}_{\text {sup }}$ a distintos tiempos y temperaturas de secado $\left(\mathrm{T} 1=75^{\circ} \mathrm{C}\right.$ a $86{ }^{\circ} \mathrm{C} ; \mathrm{T} 2=93{ }^{\circ} \mathrm{C}$ a $99{ }^{\circ} \mathrm{C} ; \mathrm{T} 3=127{ }^{\circ} \mathrm{C}$ a $\left.134{ }^{\circ} \mathrm{C}\right)$.

La Figura 5 muestra como cambió la dimensión fractal de contorno $\left(\mathrm{DF}_{\text {cont }}\right)$ con los tratamientos térmicos. Las rodajas de banana fresca se caracterizaron por presentar un contorno liso y sin alteraciones, con valores más cercanos a la unidad (forma circular). La variación de este parámetro dependió de cada tratamiento. Las muestras sometidas a condiciones de menor temperatura (T1) no mostraron cambios significativos en su contorno $(p>0,05)$, mientras que, las rodajas de banana sometidas a condiciones de temperaturas más altas (T2 y T3) presentaron bordes irregulares y sinuosos a lo largo del secado $(p<0,05)$. En el trabajo realizado por Santacruz-Vázquez et al. (2010) se evaluó el encogimiento y deformación de uvas enteras durante su deshidratación utilizando análisis fractal, y se encontró, a una mayor temperatura de secado, la formación de una costra sobre la superficie de las muestras que limita el encogimiento observandose menores valores de $\mathrm{DF}_{\text {cont }}$ (menor deformación del producto). 


\begin{tabular}{lll}
\hline & \multicolumn{1}{c}{ Temperatura } \\
\hline $\mathrm{t}=0$ min \\
$\mathrm{DF}_{\text {cont }}=1,043 \pm 0,006^{\mathrm{a}}$
\end{tabular}

Figura 5. Imágenes binarias obtenidas durante el secado y sus correspondientes valores de $\mathrm{DF}_{\text {cont. }}\left(\mathrm{T} 1=75^{\circ} \mathrm{C}\right.$ a $86{ }^{\circ} \mathrm{C}$; $\mathrm{T} 2=93{ }^{\circ} \mathrm{C}$ a $99^{\circ} \mathrm{C}$; $\mathrm{T} 3=127{ }^{\circ} \mathrm{C} \mathrm{a} 134{ }^{\circ} \mathrm{C}$ ).

El análisis de imágenes representó una alternativa por la cual fue posible monitorear la deshidratación de las rodajas de banana que se complementó con los parámetros convencionales de control, tiempo y temperatura, hasta obtener valores en donde el producto llegó a su humedad de equilibrio y en consecuencia al tiempo final de secado.

\subsection{Análisis de color}

La Figura 6A muestra que la diferencia de color $(\Delta E)$ aumentó desde el inicio del secado debido al cambio de tonalidad en la superficie de rodaja. Como un índice de evaluación importante, el color en cierta medida puede reflejar el efecto de la temperatura sobre los materiales (Romano et al., 2008). A pesar de conseguir valores similares cuando finalizó el secado, la condición de más alta temperatura (T3) mostró que el desarrollo de color fue más rápido debido al corto tiempo de procesamiento de estas muestras. Por su parte, el índice de pardeamiento $(B I)$ aumentó en función del tiempo y la temperatura de proceso (Figura 6B). El color de las rodajas de banana fue ligeramente amarillo antes del secado y oscureció después del secado (Thuwapanichayanan et al., 2011). Inicialmente este índice estuvo comprendido entre 46,02 $\pm 3,74 \mathrm{y}$ $55,54 \pm 2,56$, pero aumentó su valor ni bien comenzó la deshidratación. Al igual que con el cambio de color, la velocidad de pardeamiento del snack fue más rápida en la temperatura más alta (T3) y el comportamiento bien definido en cada una de las temperaturas guardó relación con el aumento del contraste, ya que ambos parámetros están relacionados con el oscurecimiento del material. Las rodajas de banana al final del secado tuvieron valores de $B I$ comprendidos entre $90,94 \pm 12,91$ y $105,85 \pm 3,78$.

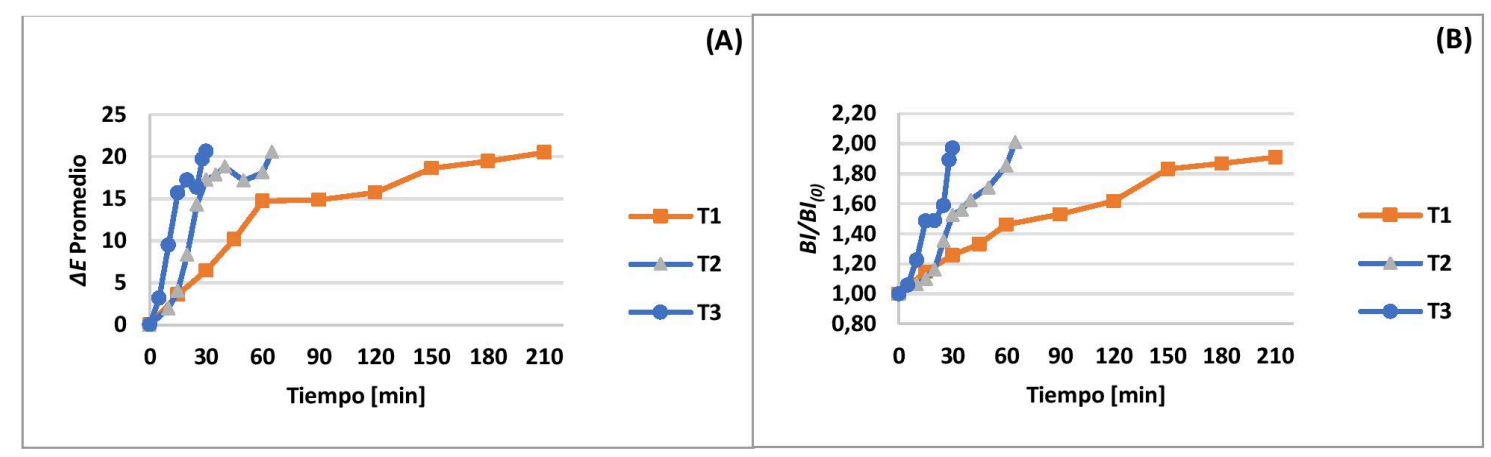

Figura 6. Efecto del proceso de deshidratación sobre los parámetros de color: (A) $\Delta E \mathrm{y}(\mathrm{B}) B I$ para las tres temperaturas de secado $\left(\mathrm{T} 1=75^{\circ} \mathrm{C}\right.$ a $86{ }^{\circ} \mathrm{C} ; \mathrm{T} 2=93{ }^{\circ} \mathrm{C}$ a $99{ }^{\circ} \mathrm{C} ; \mathrm{T} 3=127{ }^{\circ} \mathrm{C}$ a $\left.134{ }^{\circ} \mathrm{C}\right)$. 


\subsection{Microestructura del producto}

Las imágenes de rodajas de banana deshidratada presentaron una estructura porosa continua y desordenada (Figura 7). Los poros producidos fueron de forma irregular y de tamaño aleatorio, llegando a superar los 200 micrómetros en algunos casos. El desarrollo de la estructura dependió fuertemente de la temperatura de secado utilizada. Las rodajas de banana de mayor porosidad se obtuvieron a T3 mientras que las menos porosas a temperaturas de secado más bajas.
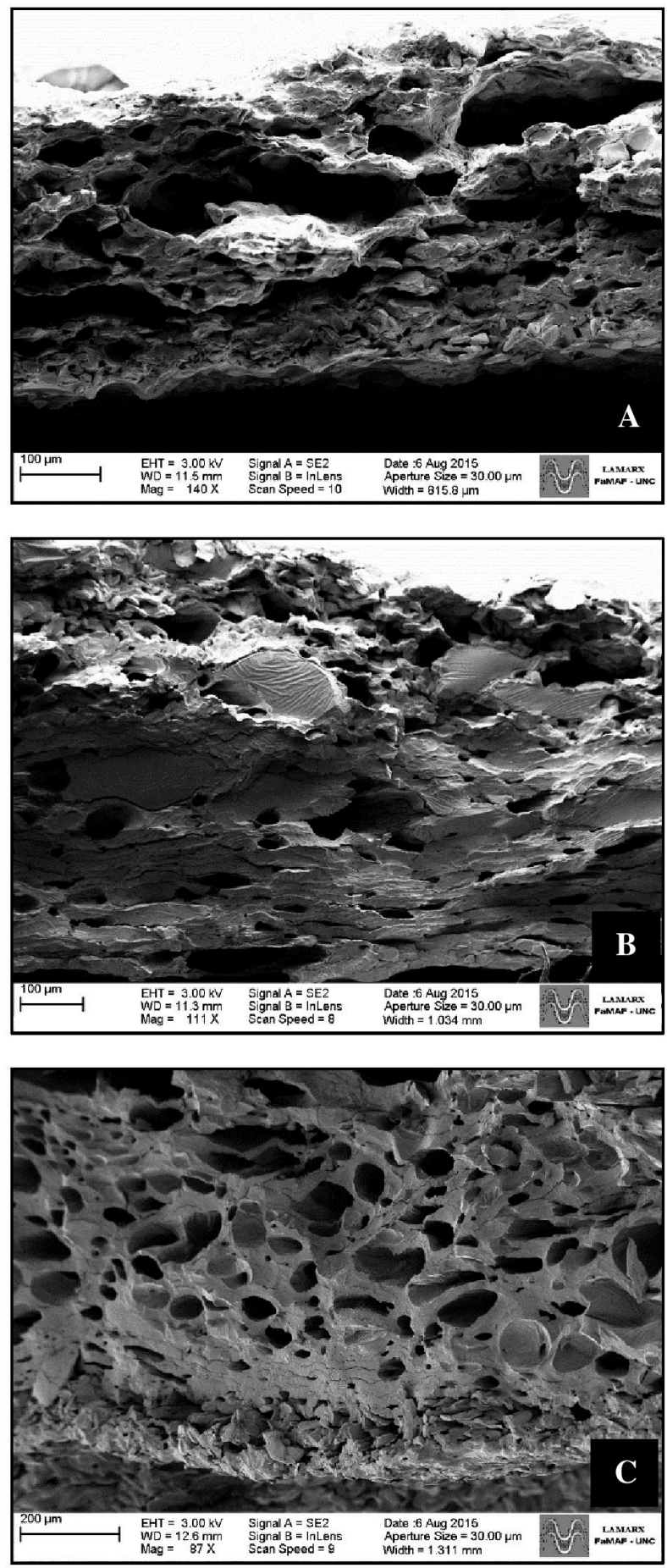

Figura 7. Micrografías SEM de los snacks de banana. (A) T1; (B) T2; (C) T3 (T1 $=75{ }^{\circ} \mathrm{C}$ a $86{ }^{\circ} \mathrm{C}$; $\mathrm{T} 2=93{ }^{\circ} \mathrm{C}$ a $99^{\circ} \mathrm{C} ; \mathrm{T} 3=127{ }^{\circ} \mathrm{C}$ a $\left.134^{\circ} \mathrm{C}\right)$. 
La migración del agua y la transferencia de calor inducen tensiones dentro de los materiales alimenticios. La formación de poros en el proceso de secado se relaciona a la producción de vapor de agua dentro de las muestras, el que produce un aumento de la presión interna y la rotura de los tejidos. La formación de poros afecta no solo las propiedades de transporte, tales como las difusividades de los gases y líquidos en las muestras, sino también la textura crujiente de las muestras, que es esencial para los productos tipo "snack" (Prachayawarakorn et al., 2008; Aprajeeta et al., 2015). Una mayor temperatura de deshidratación genera mayor gradiente de presión en el interior de la muestra y provoca un fuerte movimiento de la humedad lo que se traduce en una mayor porosidad del producto. Otros estudios han informado que mayores velocidades de secado, obtenidas por temperaturas de secado más altas, resultan en grados más altos de deformación de los productos alimenticios (Markowski et al., 2003; Prachayawarakorn et al., 2008).

\section{Conclusiones}

Los resultados indicaron que la metodología del procesamiento de imágenes es una herramienta útil que permite monitorear de forma efectiva los cambios que ocurren durante el secado convectivo de rodajas de banana. Los datos extraídos desde las imágenes, particularmente la entropía, mostraron una alta correlación con la pérdida de humedad del producto, haciendo que esta técnica sea más objetiva que la inspección visual de un operador. Esta metodología se posiciona como una alternativa que se complementa con los parámetros convencionales de tiempo y temperatura, facilitando el control del proceso, con la ventaja de ser versátil, de bajo costo y de rápida respuesta.

\section{Agradecimientos}

Los autores agradecen al Consejo Nacional de Investigaciones Científicas y Técnicas (CONICET), a la Universidad Nacional de Córdoba (UNC) y a la Agencia Nacional de Promoción Científica y Tecnológica por el apoyo financiero.

\section{Referencias}

Aprajeeta, J., Gopirajah, R., \& Anandharamakrishnan, C. (2015). Shrinkage and porosity effects on heat and mass transfer during potato drying. Journal of Food Engineering, 144, 119-128. http://dx.doi.org/10.1016/j.jfoodeng.2014.08.004

Association of Official Analytical Chemists - AOAC. (1995). Official methods of analysis of the Association of Official Analytical Chemists (16th ed.). Washington: AOAC International.

Atares, L., Sousa Gallagher, M. J., \& Oliveira, F. A. R. (2011). Process conditions effect on the quality of banana osmotically dehydrated. Journal of Food Engineering, 103(4), 401-408. http://dx.doi.org/10.1016/j.jfoodeng.2010.11.010

Baini, R., \& Langrish, T. A. G. (2007). Choosing an appropriate drying model for intermittent and continuous drying of bananas. Journal of Food Engineering, 79(1), 330-343. http://dx.doi.org/10.1016/j.jfoodeng.2006.01.068

Baini, R., \& Langrish, T. A. G. (2009). Assessment of colour development in dried bananas: Measurements and implications for modeling. Journal of Food Engineering, 93(2), 177-182. http://dx.doi.org/10.1016/j.jfoodeng.2009.01.012

Chua, K. J., Mujumdar, A. S., Hawlader, M. N. A., Chou, S. K., \& Ho, J. C. (2001). Batch drying of banana pieces: Effect of stepwise change in drying air temperature on drying kinetics and product colour. Food Research International, 34(8), 721-731. http://dx.doi.org/10.1016/S0963-9969(01)00094-1

Di Rienzo, J. A., Guzmán, A. W., \& Casanoves, F. (2002). A multiple-comparisons method based on the distribution of the root node distance of a binary tree. Journal of Agricultural, Biological and Environment Statistic, 7(2), 129-142.

Doymaz, I. (2004). Convective air drying characteristics of thin layer carrots. Journal of Food Engineering, 61(3), 359-364. http://dx.doi.org/10.1016/S0260-8774(03)00142-0

Doymaz, I. (2007). The kinetics of forced convective air-drying of pumpkin slices. Journal of Food Engineering, 79(1), 243-248. http://dx.doi.org/10.1016/j.jfoodeng.2006.01.049

Doymaz, I. (2009). Thin-layer drying of spinach leaves in a convective dryer. Journal of Food Process Engineering, 32(1), 112-125. http://dx.doi.org/10.1111/j.1745-4530.2007.00205.x

Fernández, L., Castillero, C., \& Aguilera, J. M. (2005). An application of image analysis to dehydration of apple discs. Journal of Food Engineering, 67(1-2), 185-193. http://dx.doi.org/10.1016/j.jfoodeng.2004.05.070

Gili, R., Torrez Irigoyen, M., Penci, M. C., Giner, S., \& Ribotta, P. (2018). Wheat germ thermal treatment in fluidised bed: Experimental study and mathematical modelling of the heat and mass transfer. Journal of Food Engineering, 221, 11-19.

http://dx.doi.org/10.1016/j.jfoodeng.2017.09.029 
Horuz, E., Bozkurt, H., Karataş, H., \& Maskan, M. (2017). Simultaneous application of microwave energy and hot air to whole drying process of apple slices: Drying kinetics, modeling, temperature profile and energy aspect. Heat and Mass Transfer, 54(2), 425-436. http://dx.doi.org/10.1007/s00231-017-2152-y

Leite, J. B., Mancini, M. C., \& Borges, S. V. (2007). Effect of drying temperature on the quality of dried bananas cv. prata and d'água. Food Science and Technology, 40(2), 319-323.

Markowski, M., Cenkowski, S., Hatcher, D. W., Dexter, J. E., \& Edwards, N. M. (2003). The effect of superheated-steam dehydration kinetics on textural properties of asian noodles. Transactions of the ASAE, 46(2), 389-395. http://dx.doi.org/10.13031/2013.12919

Mayor, L., \& Sereno, A. M. (2004). Modelling shrinkage during convective drying of food materials: A review. Journal of Food Engineering, 61(3), 373-386. http://dx.doi.org/10.1016/S0260-8774(03)00144-4

Mendoza, F., Dejmek, P., \& Aguilera, J. M. (2007). Colour and image texture analysis in classification of commercial potato chips. Food Research International, 40(9), 1146-1154. http://dx.doi.org/10.1016/j.foodres.2007.06.014

Monteiro, R., Carciofi, B., \& Laurindo, J. (2016). A microwave multi-flash drying process for producing crispy bananas. Journal of Food Engineering, Netherlands, 178, 1-11. http://dx.doi.org/10.1016/j.jfoodeng.2015.12.024

Organización de las Naciones Unidas para la Alimentación y la Agricultura - FAOSTAT. (2016). Base de datos sobre alimentación y agricultura: Producción de cultivos. Consultado el 30 de mayo de 2018, de http://www.fao.org/faostat/es/\#dataQC

Pan, Z., Shih, C., Mchugh, T. H., \& Hirschberg, E. (2008). Study of banana dehydration using sequential infrared radiation heating and freeze-drying. Food Science and Technology, 41(10), 1944-1951.

Park, B., \& Chen, Y. R. (2001). AE - Automation and Emerging technologies: Co-occurrence matrix texture features of multi-spectral images on poultry carcasses. Journal of Agricultural Engineering Research, 78(2), 127-139. http://dx.doi.org/10.1006/jaer.2000.0658

Prachayawarakorn, S., Tia, W., Plyto, N., \& Soponronnarit, S. (2008). Drying kinetics and quality attributes of low-fat banana slices dried at high temperature. Journal of Food Engineering, Netherlands, 85(4), 509-517. http://dx.doi.org/10.1016/j.jfoodeng.2007.08.011

Pu, Y., Zhao, M., O'Donnell, C., \& Sun, D. (2018). Nondestructive quality evaluation of banana slices during microwave vacuum drying using spectral and imaging techniques. Drying Technology, 36(13), 1542-1553. http://dx.doi.org/10.1080/07373937.2017.1415929

Quevedo, R., Mendoza, F. A., Aguilera, J. M., Chanona, J., \& Gutiérrez-López, G. (2008). Determination of senescent spotting in banana (Musa cavendish) using fractal texture Fourier image. Journal of Food Engineering, 84(4), 509-515. http://dx.doi.org/10.1016/j.jfoodeng.2007.06.013

Ranjit, K., Chethan, H., \& Naveena, C. (2016). Identification and classification of fruit diseases. International Journal of Engineering Research and Applications, 6(7), 11-14.

Rao, M. A., Rizvi, S. S. H., \& Datta, A. K. (2005). Engineering properties of foods (3rd ed., 768 p.). Boca Raton: CRC Press.

Romano, G., Baranyai, L., Gottschalk, K., \& Zude, M. (2008). An approach for monitoring the moisture content changes of drying banana slices with laser light backscattering imaging. Food and Bioprocess Technology, 1(4), 410-414. http://dx.doi.org/10.1007/s11947-008-0113-7

Santacruz-Vázquez, V., Santacruz-Vázquez, C., \& Huerta-Espinosa, V. M. (2010). Evaluación del encogimiento y deformación de uvas durante su deshidratación por fluidización usando el análisis fractal. México: Sociedad Mexicana de Ciencia y Tecnología de Superficies y Materiales.

Santacruz-Vázquez, V., Santacruz-Vázquez, C., Welti-Chanes, J., Farrera-Rebollo, R. R., Alamilla-Beltrán, L., Chanona-Pérez, J., \& Gutiérrez-López, G. F. (2008). Effects of air-drying on the shrinkage, surface temperatures and structural features of apples slabs by means of fractal analysis. Revista Mexicana de Ingeniería Química, 7(1), 55-63.

Sogi, D. S., Shivhare, U. S., Garg, S. K., \& Bawa, A. S. (2003). Water sorption isotherm and drying characteristics of tomato seeds. Biosystems Engineering, 84(3), 297-301. http://dx.doi.org/10.1016/S1537-5110(02)00275-1

Thakur, R., Pristijono, P., Bowyer, M., Singh, S., Scarlett, C., Stathopoulos, C., \& Vuong, Q. (2019). A starch edible surface coating delays banana fruit ripening. Food Science and Technology, 100, 341-347.

Thuwapanichayanan, R., Prachayawarakorn, S., Kunwisawa, J., \& Soponronnarit, S. (2011). Determination of effective moisture diffusivity and assessment of quality attributes of banana slices during drying. Food Science and Technology, 44(6), 1502-1510.

Vélez-Rivera, N., Blasco, J., Chanona-Pérez, J., Calderón-Domínguez, G., de Jesús Perea-Flores, M., Arzate-Vázquez, I., Cubero, S., \& Farrera-Rebollo, R. (2014). Computer vision system applied to classification of "Manila" Mangoes during ripening process. Food and Bioprocess Technology, 7(4), 1183-1194. http://dx.doi.org/10.1007/s11947-013-1142-4

Financiamiento: Los autores agradecen al Consejo Nacional de Investigaciones Científicas y Técnicas (CONICET), a la Universidad Nacional de Córdoba (UNC) y a la Agencia Nacional de Promoción Científica y Tecnológica por el apoyo financiero. 